Journal of Animal and Veterinary Advances 11 (13): 2323-2326, 2012

ISSN: $1680-5593$

(C) Medwell Journals, 2012

\title{
The Anatomical and Functional Outcomes of 23-Gauge Transconjunctival Sutureless Vitrectomy in Pseudophakic Retinal Detachment: A Prospective Interventional Study
}

\author{
Zhao Xiaohui, Xing Yiqiao, Li Zhi and He Tao \\ Department of Ophthalmology, Renmin Hospital of Wuhan University, Wuhan, P.R. China
}

\begin{abstract}
The purpose of this study is to evaluate the anatomic and functional outcomes of 23-gauge Transconjunctival Sutureless Vitrectomy (TSV) in primary reatment of uncomplicated pseudophakic Retinal Detachments (RD). In this prospective noncomparative interventional study, 32 eyes of 32 patients with $\mathrm{RD}$ after cataract surgery with phacoemulsification were evaluated. Primary pseudophakic RDs with macular detachment and proliferative vitreoretinopathy stage B or less were included in the study. The 23-gauge TSV, perfluorocarbon liquid injection followed by air exchange, endolaser photocoagulation and sulfur hexafluoride gas (20\%) injection were applied to all eyes. Mean follow-up time was 9.3 months (range, 6-12 months). Retinal reattachment with a single operation was achieved in $93.75 \%$ of eyes and with additional surgery, the retina was reattached in $100 \%$ of eyes. Preoperative visual acuity was $<20 / 200$ in all eyes (range, hand motions to $20 / 400$ ). Postoperative visual acuity was 20/40 or better in 15 eyes (46.88\%) and 20/50 and 20/200 in 12 eyes (37.50\%). No sutures were required to close the scleral and conjunctival openings. Postoperative complications were Transient hypotony in 2 eyes (6.25\%) macular pucker in 2 eyes $(6.25 \%)$ and cystoid macular edema in 2 eyes $(6.25 \%)$.
\end{abstract}

Key words: 23 -gauge, transconjunctival sutureless vitrectomy, pseudophakic retinal detachment, sulfur, hexafluride gas, air exchange

\section{INTRODUCTION}

Retinal Detachment ( $\mathrm{RD}$ ) is one of the serious complications of cataract surgery that threatens visual acuity and occurs in approximately $0.4-1.0 \%$ of eyes after cataract surgery with phaco emulsification (Haddad et al., 2002; Olsen and Olson, 2000). The 20-gauge Primary Pars Plana Vitrectomy (PPV) with or without scleral buckling are the most commonly used techniques in the treatment of primary pseudophakic RD (Lois and Wong, 2003; Stangos et al., 2004). The advent of new microsurgical instruments, wide-angle viewing apparatus and Perfluorocarbon Liquids (PFCL) have increased the scope of primary PPV in the treatment of pseudophakic RD.

The recent evolution in the PPV technique with minimal invasive surgery and faster patient recovery is 23-gauge Transconjunctival Sutureless Vitrectomy (TSV) (Eckart, 2005). Several researchers have reported successful anatomic and visual outcomes with 23-gauge TSV in eyes with different vitreoretinal disorders (Sanisoglu et al., 2011; Romano et al., 2012; Wu et al., 2011). However, there is limited experience in pseudophakic RD. The purpose of this study was to report the preliminary results of 23-gauge TSV in the management of primary pseudophakic $\mathrm{RD}$.

\section{MATERIALS AND METHODS}

The 32 consecutive patients ( 32 eyes) were recruited in the period between Feburary, 2007 and May, 2011 in the Department of Ophthalmology at Renmin Hospital of Wuhan University. All patients suffered retinal detachment after cataract surgery with implantation of an intraocular lens. Inclusion criteria were RD occurring after cataract extraction with phacoemulsification and posterior chamber Intraocular Lens (IOL) implantation; macular detachment; Proliferative Vitreoretinopathy (PVR) grade A or B (Anonymous, 1983). The patients with signs of PVR higher than grade $\mathrm{B}$, proliferative diabetic retinopathy, uncontrolledv glaucoma or uveitis, vitreous bleeding, giant tear, anterior chamber IOL, presence of posteriorly dislocated lens fragments, a history of any other intraocular surgery except cataract extraction were excluded. Eyes that had been operated due to traumatic and congenital cataracts were excluded as well. The possible merits and risks of the treatment were explained to the patients and an informed consent was obtained in accordance with the Helsinki Declaration prior to inclusion in the study.

Preoperative evaluation included age, sex, duration of macular detachment best-corrected Snellen visual acuity,

Corresponding Author: Xing Yiqiao, Department of Ophthalmology, Renmin Hospital of Wuhan University, No. 238 Jiefang Road, Wuchang District, 430060 Wuhan, Hubei Province, China 
slit-lamp biomicroscopy examination Intraocular Pressure (IOP) and detailed fundus examination. Axial length and refractive status measurements were obtained from the patients' medical records.

All operations were performed by an experienced surgeon under local anesthesia. The microcannulae (DORC International, Zuidland, Holland) were inserted obliquely with the help of the insertion trocars in the inferotemporal, superotemporal and superonasal quadrants. The overlying conjunctiva was pulled aside with forceps during the entrance of the insertion trocar in order to avoid the alignment between conjunctival and scleral incisions. The infusion cannula was placed in the inferotemporal quadrant and plugs were used to temporarily close the other entry sites. A cutting rate of 1500 cuts $\mathrm{min}^{-1}$ and a vacuum level of $450 \mathrm{mmHg}$ were used during PPV. The bottle height was set at $40 \mathrm{~cm}$. Peripheral Vitreous was removed during PPV. Peripheral manipulations were performed by using wide-angle viewing system with scleral depression by an assistant. Following PPV, the infusion line was removed while the microcannula was held in place with forceps. A PFCL (Perfluoronoctane) was then injected slowly up to the anterior break to flatten the retina. The infusion line was replaced and switched to air to perform a complete PFCL-air exchange.

A viscoelastic solution (Sodium Hyaluronate 1\%) was injected into the anterior chamber through a paracentesis to avoid air passage to the anterior chamber. Endolaser photocoagulation ( $532 \mathrm{~nm}$ ) was applied around the retinal break (s) and to the degenerative retina area. Then the superonasal microcannula was removed and sulfur hexafluoride gas (20\%) was injected through the infusion line. After removing the superotemporal microcannula, the inferotemporal microcannula and infusion line were removed en bloc while injecting gas. The conjunctiva overlying the sclerotomy was slightly displaced to disrupt the alignment between both entry sites and an antibiotic and corticosteroid combination was injected into the subconjunctival space. The viscoelastic solution was left in the anterior chamber at the end of the operation. All patients were postured face down for $3 \mathrm{~h}$ postoperatively thereafter the patients were postured on the opposite side of the breaks. Posturing was maintained for 7-10 days. Patients were examined daily until discharge and thereafter 1,3 and 6 months and 1 year after surgery. Each postoperative examination included measurements of best-corrected Snellen visual acuity, slit-lamp examination Intraocular Pressure (IOP) and fundus examination. Main outcome measures were retinal reattachment rate, visual acuity Intraocular Pressure (IOP) and intraoperative and postoperative complications.

\section{RESULTS AND DISCUSSION}

Of 32 patients, 15 were men and 17 were women. Mean age was 62.32 years (range, 28-72 years). Mean follow-up time was 9.3 months (range, 6-12 months). Preoperative characteristics including intraocular lens, refractive status, vitrous loss or laser capsulotomy and detection of hole of patients are shown in Table 1. Preoperative visual acuity was Hand Motions (HM) in 25 eyes (78.13\%), 20/400 in 5 eyes (15.63\%) and 20/800 in 2 eyes $(6.25 \%)$. Mean duration of macular detachment was 7.1 days (range, 1-23 days). Anterior vitrectomy with sulcus-fixated IOL implantation had been performed in 5 eyes $(15.63 \%)$ with posterior capsule rupture and vitreous loss complicating the phacoemulsification procedure. About 11 eyes (34.38\%) with posterior capsule opacity had neodymium:YAG (Nd:YAG) laser capsulotomy. The remaining 9 eyes $(50.00 \%)$ had intact posterior capsule. Mean axial length was $25.5 \pm 2.3 \mathrm{~mm}$ and 4 eyes had pathologic myopia with an axial length of 29 and $30 \mathrm{~mm}$. Single operation success was achieved in $30(93.75 \%)$ of 32 eyes. In 1 eye, retinal redetachment due to a new inferior break which was observed at postoperative 3 weeks was successfully treated with reoperation using retinocryopexy and scleral buckling. In a second patient redetachment was associated with the development of PVR with reopening of the previous retinal break 28 days after primary vitrectomy. In a second procedure, researchers removed these epiretinal membranes by 23-gauge TSV system. The retina reattached after injection of perfluorocarbon liquids, endolaser photocoagulation and fluid gas exchange.

The median of best corrected visual acuity before surgery was $20 / 100$. Owing to the gas endotamponade median visual acuity decreased to $20 / 400$ at the time of discharge. At the 1 month interval, the gas endotamponade was totally absorbed in all cases. Median visual acuity increased to $20 / 80$. After 6 months, median visual acuity was $20 / 40$ but 4 patients did not missed the control examination at this interval. Median visual acuity 1 year after primary vitrectomy was 20/30.

Table 1: Characteristics of 32 pseudophakic eyes with retinal detachment

\begin{tabular}{lc}
\hline Characteristics & No. of eyes \\
\hline Posterior intraocular lens & 32 \\
Vitreous loss & 5 \\
YAG capsulotomy & 11 \\
Myopia(>-6.0D) & 14 \\
Detection of hole & \\
No hole of preoperatively & 7 \\
No hole according to Lincoff preoperatively & 6 \\
No hole intraoperatively & 2 \\
A additional hole intaoperatively & 5 \\
\hline
\end{tabular}


Final visual acuity was better than preoperative visual acuity in all eyes. Postoperative visual acuity was $20 / 40$ or better in 15 eyes ( $46.88 \%$ ) and between $20 / 50$ and $20 / 200$ in 12 eyes (37.50\%). Mean IOP was $11.43 \mathrm{~mm} \mathrm{Hg}$ (range, 7-17) before the operation, $11.8 \mathrm{~mm} \mathrm{Hg}$ (range, 8-21) at day and $17.1 \mathrm{~mm} \mathrm{Hg}$ (range, 10-27) at month 1 after the operation.

In 7 eyes no breaks were detected preoperatively. In a further 6 eyes preoperative localisation of the break did not comply with the rules of Lincoff (Lincoff and Gieser, 1971) but corresponding breaks were found intraoperatively. In all but 2 eyes vitrectomy and the use of perfluorocarbon liquid revealed the causative retinal break. In a further 5 eyes an additional break not seen in the preoperative examination could be identified. No major intraoperative complications were encountered and conversion to conventional 20-gauge PPV was not required in any eye. Intraocular manipulations were easily performed and no sutures were required to close the scleral and conjunctival openings. No clinically significant intraocular inflammation was observed in the postoperative period due to the viscoelastic solution left in the anterior chamber. Postoperative complications were transient hypotony in 2 eyes $(6.25 \%)$, macular pucker in 2 eyes $(6.25 \%)$ and cystoid macular edema in 2 eyes $(6.25 \%)$.

Pars plana vitrectomy offers a number of advantages to the retinal surgeon in the management of pseudophakic retinal detachments (Bartz-Schmidt et al., 1996). It eliminates media opacities in the anterior and posterior segment. It allows for easy localisation of retinal holes and removalof vitreoretinal traction and it facilitates more precise treatment of retinal breaks by endolaser. Recently, the new advance in the PPV technique with minimal invasive surgery and faster patient recovery is 23-gauge Transconjunctival Sutureless Vitrectomy (TSV) (Eckart, 2005). Several researchers have reported successful anatomic and visual outcomes with 23-gauge TSV in eyes with different vitreoretinal disorders (Sanisoglu et al., 2011; Romano et al., 2012; Wu et al., 2011). However, there is limited experience in pseudophakic RD with this new technique.

In this prospective study, retinal reattachment with a single operation using 23-gauge TSV was achieved in $93.75 \%$ of eyes with pseudophakic $\mathrm{RD}$ and with additional surgery, the retina was reattached in $100 \%$ of eyes. Single operation and final anatomic success with primary 20 -gauge PPV have been reported in $63-98$ and $92-100 \%$ of eyes with pseudophakic RD, respectively (Stangos et al., 2004; Campo et al., 1999; Ahmadieh et al., 2005). In addition, the single operation and final success rates compare favorably with the anatomic outcomes of other surgical procedures used in the management of pseudophakic RD (Stangos et al., 2004; Campo et al.,
1999; Tornambe et al., 1991). Therefore, the preliminary results suggest that 23 -gauge TSV may be an alternative surgical procedure in the treatment of selected cases of pseudophakic RD.

In the present study, final visual acuity was better than preoperative visual acuity in all eyes. Postoperative visual acuity was $20 / 40$ or better in 15 eyes (46.88\%) and between $20 / 50$ and $20 / 200$ in 12 eyes (37.50\%). In previous studies in which primary 20 -gauge PPV was performed in the treatment of pseudophakic RD visual acuity of 20/40 or better has been reported in 11-88\% of eyes (Lois and Wong, 2003; Campo et al., 1999; Ahmadieh et al., 2005; Brazitikos et al., 2005). The visual results are also comparable with the visual outcomes of other surgical procedures used in the management of pseudophakic RD (Campo et al., 1999; Ahmadieh et al., 2005; Brazitikos et al., 2005). There are several advantages of 23-gauge TSV over conventional 20-gauge PPV including less operation time, absence of suture-related irritation or local inflammatory reaction at the sclerotomy sites, absence of limbal stem cell damage due to conjunctival dissection insignificant corneal surface and astigmatic changes in the early postoperative period, faster postoperative recovery and increased patient comfort (Sanisoglu et al., 2011; Romano et al., 2012; Wu et al., 2011). In addition, the potential advantages of 20-gauge PPV without scleral buckling over conventional scleral buckling are expected to be the advantages of 23-gauge TSV. These are less operation time, accurate diagnosis of breaks controlled drainage of subretinal fluid and no postoperative axial length changes. Therefore if a primary PPV is appropriate in the management of pseudophakic $\mathrm{RD}$, it may be logical to perform it with a 23 -gauge TSV system.

Anterior or posterior capsular fibrosis small capsulorrhexis, cortical remnants, poor pupillary dilation, small IOL diameter, formation of moisture droplets on the posterior surface of the IOL and optical aberrations secondary to IOL itself make the PPV more difficult in pseudophakic eyes when compared with the primary RD (Yoshida et al., 1992). The indication of 23-gauge TSV can be extended to pseudophakic $\mathrm{RD}$ and favorable outcomes may be obtained by paying special attention to the above issues. The possible risks of the TSV system were postoperative vitreous incarceration leading to anterior vitreoretinal traction and late period retinal tear or detachment, endophthalmitis and hypotony. Postoperative $\mathrm{RD}$, transient or severe hypotony, shallow choroidal detachment, endophthalmitis have been reported in studies in which 23-gauge TSV was performed for different vitreoretinal disorders (Romano et al., 2012; Cekic et al., 2011; Kim et al., 2007). Retinal redetachment $(6.25 \%)$ due to a new break, transient hypotony $(6.25 \%)$ macular pucker $(6.25 \%)$ and cystoid macular edema 
$(6.25 \%)$ postoperative complications encountered in the series. Despite peripheral vitreous removal which increases the risk of postoperative hypotony, an IOP of $<5 \mathrm{~mm} \mathrm{Hg}$ and choroidal detachment were not observed in any eye. This may be due to the injection of a viscoelastic solution (sodium hyaluronate $1 \%$ in the series) into the anterior chamber and also, due to the use of intravitreal gas injection. Residual peripheral vitreous which is pushed towards the sclerotomies by intravitreal gas may serve as a plug and prevent leakage even in eyes with peripheral vitreous removal. The primary limitations of this study are the small number of patients, selection of cases without severe PVR and lack of comparison with other surgical procedures used in the management of pseudophakic RD. In conclusion, primary 23-gauge TSV system appears to be effective and safe in the management of uncomplicated pseudophakic RD. Faster postoperative recovery and increased patient comfort make this surgical procedure a good alternative to other procedures used in the management of pseudophakic RD.

\section{CONCLUSION}

In this study, 23-gauge sutureless vitrectomy resulted in higher single surgery and final anatomic success rates in primary treatment of noncomplicated RDs in pseudophakic eyes with commensurate increase in visual acuity. Primary 23-gauge TSV system appears to be an effective and safe procedure in the treatment of uncomplicated pseudophakic RD.

\section{REFERENCES}

Ahmadieh, H., S. Moradian, H. Faghihi, M.M. Parvaresh and $\mathrm{H}$. Ghanbari et al., 2005. Anatomic and visual outcomes of scleral buckling versus primary vitrectomy in pseudophakic and aphakic retinal detachment: Six-month follow-up results of a single operation-report No. 1. Ophthalmology, 112: 1421-1429.

Anonymous, 1983. The classification of retinal detachment with proliferative vitreoretinopathy. Ophthalmology, 90: 121-125.

Bartz-Schmidt, K.U., B. Kirchhof and K. Heimann, 1996. Primary vitrectomy for pseudophakic retinal detachment. Br. J. Ophthalmol., 80: 346-349.

Brazitikos, P.D., S. Androudi, W.G. Christen and N.T. Stangos, 2005. Primary pars plana vitrectomy versus scleral buckle surgery for the treatment of pseudophakic retinal detachment: A randomized clinical trial. Retina, 25: 957-964.
Campo, R.V., J.O. Sipperley, S.R. Sneed, D.W. Park, P.U. Dugel, J. Jacobsen and R.J. Findall, 1999. Pars plana vitrectomy without scleral buckle for pseudophakic retinal detachments. Ophthalmology, 106: 1811-1815.

Cekic, O., M. Cakir, S. Yazgan and O.F. Yilmaz, 2011. Acute endophthalmitis following 23-gauge sutureless transconjunctival vitrectomy. Indian J. Ophthalmol., 59: 160-162.

Eckart, C., 2005. Transconjunctival sutureless 23-gauge vitrectomy. Retina, 25: 208-211.

Haddad, W.M., C. Monin, C. Morel, P. Larricart and S. Quesnot et al., 2002. Retinal detachment after phacoemulsification: A study of 114 cases. Am. J. Ophthalmol., 133: 630-638.

Kim, M.J., K.H. Park, J.M. Hwang, H.G. Yu, Y.S. Yu and H. Chung, 2007. The safety and efficacy of transconjunctival sutureless 23-gauge vitrectomy. Korean J. Ophthalmol., 21: 201-207.

Lincoff, H. and R. Gieser, 1971. Finding the retinal hole. Arch. Ophthalmol., 85: 565-569.

Lois, N. and D. Wong, 2003. Pseudophakic retinal detachment. Surv. Ophthalmol., 48: 467-487.

Olsen, G. and R.J. Olson, 2000. Update on a long-term, prospective study of capsulotomy and retinal detachment rates after cataract surgery. J. Cataract Refract. Surg., 26: 1017-1021.

Romano, M.R., R. Das, C. Groenwald, T. Stappler and J. Marticorena et al., 2012. Primary 23-gauge sutureless vitrectomy for rhegmatogenous retinal detachment. Indian J. Ophthalmol., 60: 29-33.

Sanisoglu, H., M.S. Sevim, B. Aktas, S. Sevim and A. Nohutcu, 2011. Outcomes of 23-gauge pars plana vitrectomy and internal limiting membrane peeling with brilliant blue in macular hole. Clin Ophthalmol., 5: 1177-1183.

Stangos, A.N., I.K. Petropoulos, C.G. Brozou, A.D. Kapetanios and A. Whatham et al., 2004. Pars-plana vitrectomy alone vs vitrectomy with scleral buckling for primary rhegmatogenous pseudophakic retinal detachment. Am. J. Ophthalmol., 138: 952-958.

Tornambe, P.E., G.F. Hilton, D.A. Brinton, T.P. Flood and S. Green et al., 1991. Pneumatic retinopexy. A twoyear follow-up study of the multicenter clinical trial comparing pneumatic retinopexy with scleral buckling. Ophthalmology, 98: 1115-1123.

Wu, W.C., C.C. Lai, R.I. Lin, N.K. Wang and A.N. Chao et al., 2011. Modified 23-gauge vitrectomy system for stage 4 retinopathy of prematurity. Arch. Ophthalmol., 129: 1326-1331.

Yoshida, A., H. Ogasawara, A.E. Jalkh, R.J. Sanders and J.W. McMeel et al., 1992. Retinal detachment after cataract surgery. Predisposing factors. Ophthalmology, 99: 453-459. 